\title{
NOVEMBRE 1972
}

54. ANNÉE - N•647

\section{revue internationale de la croix-rouge}

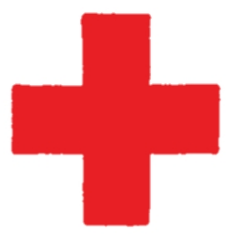

INTER ARMA CARITAS

GENÈVE

COMITE INTERNATIONAL DE LA CROIX-ROUGE FONDÉ EN 1863 


\section{COMITÉ INTERNATIONAL DE LA CROIX-ROUGE}

MM. MARCEL A. NAVILLE, president (membre depuis 1967)

JEAN PICTET, docteur en droit, président de la Commission juridique, vice-president (1967)

HARALD HUBER, docteur en droit, juge fédéral, vice-président (1969)

PAUL RUEGGER, ambassadeur, président du CICR de 1948 à 1955 (1948)

GUILLAUME BORDIER, ingénieur dipl. E.P.F., M.B.A. Harvard, banquier (1955)

HANS BACHMANN, docteur en droit, Stadtrat de Winterthour (1958)

JACQUES FREYMOND, docteur ès lettres, directeur de l'Institut universitaire des hautes études internationales, professeur à l'Université de Genève (1959)

DIETRICH SCHINDLER, docteur en droit, professeur à l'Université de Zurich (1961)

$M^{11}$ MARJORIE DUVILLARD, infirmière (1961)

MM. MAX PETITPIERRE, docteur en droit, ancien président de la Confédération suisse (1961)

ADOLPHE GRAEDEL, député au Conseil national suisse de 1951 à 1963, ancien secrétaire génćral de la Fédération internationale des ouvriers sur métaux (1965)

$M^{\text {me }}$ DENISE BINDSCHEDLER-ROBERT, docteur en droit, professeur à l'Institut universitaire des hautes études internationales (1967)

MM. JACQUES F. DE ROUGEMONT, docteur en médecine (1967)

ROGER GALLOPIN, docteur en droit, ancien directeur général (1967)

WALDEMAR JUCKER, docteur en droit, secrétaire de l'Union syndicale suisse (1967)

VICTOR H. UMBRICHT, docteur en droit, administrateur (1970), en congé

PIERRE MICHELI (1971)

Membres honoraires: M. JACQUES CHENEVIERE, vice-president d'honneur; $\mathrm{M}^{1 \mathrm{ll}}$ LUCIE ODIER, vice-presidente d'honneur; MM. CARL J. BURCKHARDT, PAUL CARRY, $M^{\text {me }}$ MARGUERITE GAUTIER-VAN BERCHEM, MM. SAMUEL A. GONARD, EDOUARD DE HALlER, PAUl LOGOZ, RODOLFO OLGIATI, FREDÉRIC SIORDET, ALFREDO VANNOTTI, ADOLF VISCHER.

Direction: MM. JEAN-LOUIS LE FORT, secrétaire général;

CLAUDE PILLOUD, directeur du département des principes et du droit;

JEAN-PIERRE MAUNOIR, sous-directeur, chargé ad interim de la direction du département des opérations. 


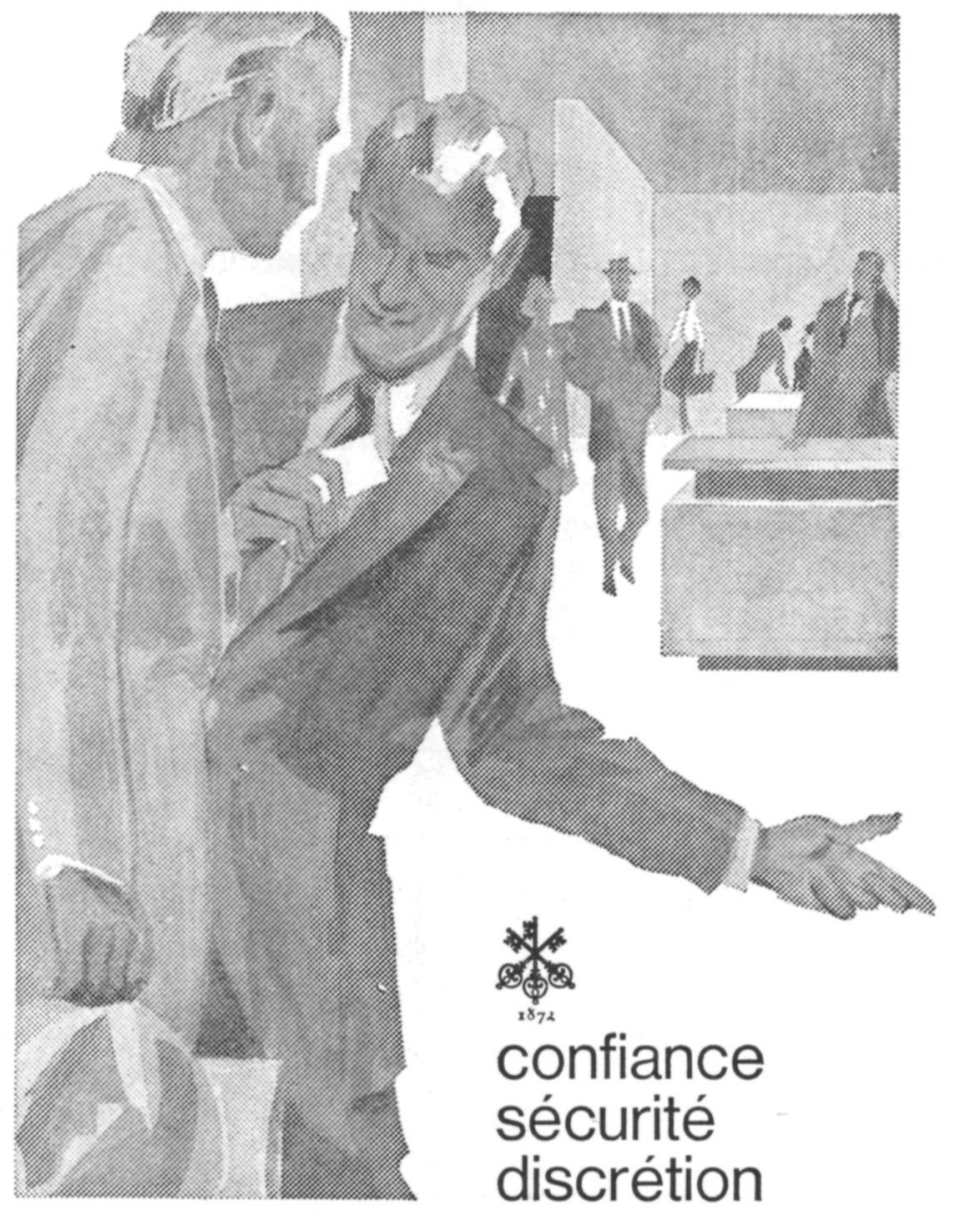

\section{SOCIÉTÉ DE BANQUE SUISSE SWISS BANK CORPORATION}

Siège de Genève: 2, rue de la Confédération Tél. 266211 


\section{SOCIETE FIDUCIAIRE ROMANDE OFOR S.A.}

Place Saint-Gervais 1, Genève - Tél. 317050

— Expertises, revisions et organisations comptables

\ Domiciliation et administration de sociétés

$\downarrow$ Assainissements et liquidations

Interventions et conseils en matière fiscale

\section{JOURVAL DR GEIEVVE}

deux imprimeries

deux adresses

rue François-Dussaud 20

1211 Genève 8 - Téléphone 431940

rue Général-Dufour 5-7

1211 Genève 11 - Téléphone 250350

TYPO - OFFSET - ROTATIVE 


\section{SOMMAIRE}

COMITE INTERNATIONAL DE LA CROIX-ROUGE

DANS LE MONDE DE LA CROIX-ROUGE

FAITS ET DOCUMENTS

LIVRES ET REVUES

\section{REVUE INTERNATIONALE DE LA CROIX-ROUGE}

NOVEMBRE 1972 - №647

Paul Weis : Les réfugiés et les droits de l'homme (II)

$K$. Pétrov et $D$. Vénov : Sauvetage en montagne et sauvetage nautique en Bulgarie

Activités extérieures:

Le Président du CICR en République démocratique allemande - Sous-continent asiatique République khmère - Laos - République du Vietnam - Mission du délégué général en Afrique - Ouganda - Moyen-Orient - République dominicaine - Paraguay - Uruguay .

Le CICR inaugure une nouvelle exposition itinérante

Le développement de la station radio du CICR . 692 Activités du Service des secours . . . . . . . . 696

Ligue des Sociétés de la Croix-Rouge . . . . . . 698

Une édition anglaise du "Manuel de la Croix-

Rouge internationale » . . . . . . . . . 701

Le monde a-t-il encore besoin de nous ? . . . . 702 France . . . . . . . . . . . . . 704

Kenya . . . . . . . . . . . . 705

$\mathrm{VI}^{\mathrm{e}}$ Cours international de perfectionnement pour jeunes médecins militaires . . . . . . 706

Conférence internationale de l'action sociale . . 708 
INTERNATIONAL

REVIEW

OF THE RED CROSS

\section{SUPPLEMENTS}

DE LA REVUE

EN LANGUE ESPAGNOLE

EN LANGUE ALLEMANDE

REVUE INTERNATIONALE DE LA CROIX-ROUGE
Une édition en langue anglaise paraît chaque mois. Elle est en principe identique à l'édition française, et peut être obtenue aux mémes conditions.

Frédéric de Mulinen: Señalamiento e identificación del personal y material sanitarios.

Sachiko Hashimoto: Das Rote Kreuz und die Jugenderziehung - Funkdienst des IKRK - Das Soldatenhandbuch - Der Präsident des IKRK in der Deutschen Demokratischen Republik.

Elle est publiée chaque mois par le Comité international de la Croix-Rouge.

7, avenue de la Paix, 1211 Genève 1 (Suisse) - Compte de chèques postaux $12-1767$.

Abonnement un an: Fr. 30,-; le numéro: Fr. 3,-.

RÉDACTION : J.-G. LOSSIER

Seuls les textes signés par le Comité international de la Croix-Rouge engagent la responsabilité de celui-ci. 